\title{
Computer control of teaching enhancement by communication networked
}

\author{
Nada N. Tawfeeq ${ }^{1}$, Mehdi J. Marie ${ }^{2}$, Khalaf S Gaeid ${ }^{3}$ \\ ${ }^{1.3}$ Electrical Engineering Department, Tikrit University, Iraq \\ ${ }^{2}$ Computer Science and Information Technology, Kirkuk University, Iraq
}

\begin{tabular}{l} 
Article Info \\
\hline Article history: \\
Received Jul 19, 2019 \\
Revised Oct 20, 2019 \\
Accepted Nov 10, 2019 \\
\hline Keywords: \\
E-learning \\
Teaching enhancement \\
Wireless networked control \\
systems \\
WSN \\
ZigBee
\end{tabular}

Mehdi J. Marie,

Alzawrah Industrial Co.,

Baghdad/Iraq.

Email: mehdijelo@gmail.com

\begin{abstract}
Enhancement of teaching and education is crucial. This enhancement is rapidly improved through combining communication and technology resulting in various benefits (particularly the wireless Technologies (WT)). Such combination encourages lecturers to meet the worldwide demand for changing traditional teaching techniques to technology-based methods. Wireless system networks (WSNs) represent systems of smart devices that are linked wirelessly. In addition, these devices are characterized by having a bandwidth of bounded communication, abilities of sensing and computation. For performing operations of control, this system employs a Flywheel Position Control System (FW PCS) as a plant node. This study utilizes the True Time 1.5 MATLAB/Simulink platform for simulating and implementing the WSN mathematical model with wireless networks. The simulation is carried out between plant nodes and controller using the IEEE 802.15.4 standard (also called ZigBee) via protocols of Wi-Fi. The suggested E-learning model comprises a set of properties that can encourage and enhance the level of students in various learning purposes and stages.
\end{abstract}

Copyright $\odot 2020$ Institute of Advanced Engineering and Science. All rights reserved.

\section{INTRODUCTION}

In education, resources and innovative implementations ways, facilitation, growth and designing are provided by technology. Any initiative will be successful through employing technology in a learning program that is based on the involved users' attitudes and support [1]. Technology is implemented by instructors in constantly varying environments of education. Technology can change the method of teaching and learning. In the case of language learning, various educational technologies (such as interactive whiteboards, robots, desktop computers, laptops and smartphones) can be used [2]. Recently, wireless communication and networking courses become increasingly popular in educational institutions such as postsecondary colleges, universities, technical institutions and institutions of private training worldwide [3].

The literature is rich in works with several merits of the real-time social networking employed for different purposes such as educational programs. The growing communication and interaction among students which supports experience and learning motivation, as well as providing materials of personalized course can be attributed to the issues brought to students [4].

In the last decade, communication, control engineering and computer have witnessed enormous progress. By utilizing wireless transmission, tiny cheap devices have been developed and enhanced by the sensing capability, data collection, in addition to communicating with other devices. Currently, in WSN, equipment is available for improving intelligent, complex systems on the basis of these devices with simplicity and efficiency. 
In control engineering literature, Closed-Loop Networked Control Systems (CLNCSs) are classified as spatially distributed control systems. In these systems, sensors, controllers and actuator communicate among themselves over a shared network of band-bounded digital communication. The communication occurs through utilizing wired line or criteria of wireless communication [5]. Traditionally, CLNCSs" are widely employed for decreasing cost and weight, as well as for increasing reliability and connectivity. During the last decades, different types of industrial networks of communication have been improved [6]. Examples of such networks are the Distributed Control System (DSC) and Supervisory Control and Data Acquisition systems (SCADA) [7-8]. In industry, employing WT has several advantages like scalability, flexibility, reliability and cost-effectiveness. Three technologies are incorporated in Wireless Networked Control Systems" (WNCSs) based on Wireless Sensor Networks (WSNs), they are wireless communication, computer networks and control engineering.

Presently, a considerable development is seen in wireless networks in various fields and applications. The main positive features of WT include effectiveness, mobility, cost competition and configurable. Though WT was firstly used for communication and sensing through WSNs, a new field has now arisen based on utilizing the exact techniques for authorizing network control systems [9].

The remainder of this paper is organized as follows. Necessary information on WNCS applications is introduced in Section II. Computer Science Framework features are presented in Section III. Then, wireless automatic control systems characteristics are described in Section IV. The experimental setup is explained in Section V. Section VI evaluates the effectiveness of the Wi-Fi project. Finally, Section VII concludes this article.

\section{WNCSS APPLICATIONS AND FRAMEWORK}

In the research field of WNCSs, three main areas can be identified [10], as follows:

a. Control of networks: this area deals with the design of wireless networks system and strategies of control.

b. Research of networks: it studies the application of networks topology and protocols of communication for real-time CLNCSs implementation.

c. Multi-agent system: it examines the effect of interactions between both network's components and architectures on the control's global goals.

Recently, a multi-hop induced gain scheduler was proposed for WNCS [11]. The architecture of client-server represented the basis of the proposed scheme of control. On the other hand, the authors in [12] presented modeling accession for NCSs with a number of patterns of changing periods of communication latency.

Experimental optimization of a wireless transmission scheme has suggested in [13]. They also investigated a tuning process of the controller parameters for applications of real-time control. An investigation on WNCS with varying routes of time delay was conducted in [14]. To recompense the loss, the study examined on a packet-based network where posterior packets catch up the prior packets. The authors in [15] investigated the overall architecture of SCADA and WSNs systems and commonly adopted communication protocols. The XBee platform has been utilized as a case study. Model of predictive control was used for controlling the unmanned quadrotor helicopters attitude for wireless transmission and image compression [16]. Using an XBee modem, a wireless PLC system was proposed to control the distant domain equipments without wiring to construct the wireless network between controller (i.e. the master station) and plants (i.e. the remote devices) [17].

The authors in [18] developed an experimental validation and layout of predictive control model of hybrid dynamical processes with wireless sensors. Early in this decade, the authors in [19] studied the performance analysis and delays under packet loss of the PIDPLUS controller versus PID and PI controllers. One year later, the significant problems of wireless networked control systems were examined by studying the influence of the wireless network on control execution. Additionally, the authors developed a close design method to attain the required cost of control while decreasing the network energy consumption. The study of [21] has developed an Internet-based DSC system for controlling oil refineries. This system comprises some WT as part of the system. In [22], Lemmon defined the proper terms for the NCSs stability under random drop-outs.

Over a wireless network, packet loss compensation technique was proposed in [23] for cyberphysical control. It is well known that the Zigbee protocol is usually used as a communication network and wireless medium access. In addition, the authors in [24] have addressed the stability issue for WNCSs with impulse disturbance in the discrete time domain. Furthermore, in [25], using an NCS method, the authors explained the utilization of ZigBee (or IEEE 802.15.4) as a communication protocol. This method has called as the wireless model based predictive networked control system. The authors also described the 
implementation of a control system. However, new challenges have emerged in terms of how to improve accuracy, quality of services (QoS) and reliability [26].

\section{COMPUTER SCIENCE FRAMEWORK}

Incorporating of Information, Communication and Technology (ICT) in education denotes the steps of teaching and learning that use technology related to implementing technologies of teaching in schools. Nowadays, it is seen that most of the students have better knowledge within the technology-based environment, so there is a vital problem represented by implementing ICT in schools' classrooms [27]. Administrators at levels of district and school can contribute to successful pursuit and application of educational technology initiatives as shown in Figure 1.

Recently, standards have developed by the International Society for Technology in Education (ISTE) aim to guide the responsibilities of a technology coach. Therefore, as a specialist in applying and demonstrating these standards, it is a duty to assist and support all stakeholders to develop a technology-rich professional learning program [28].

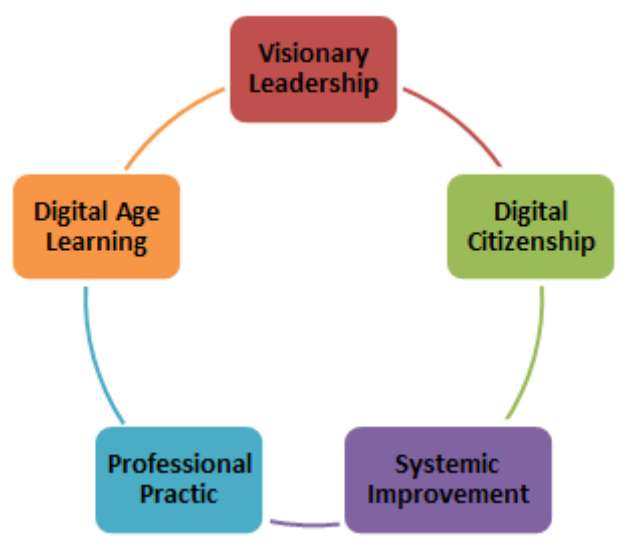

Figure 1. The administrators in learning

\section{WIRELESS AUTOMATIC CONTROL SYSTEM}

As shown in Figure 2, WNCS should be well-designed and amenable enough to be able to control all its actions. From an engineering perspective, it also should have the ability to perform the wanted assignments correctly and precisely. However, there are other definite characteristics that a WNCS must have [29].

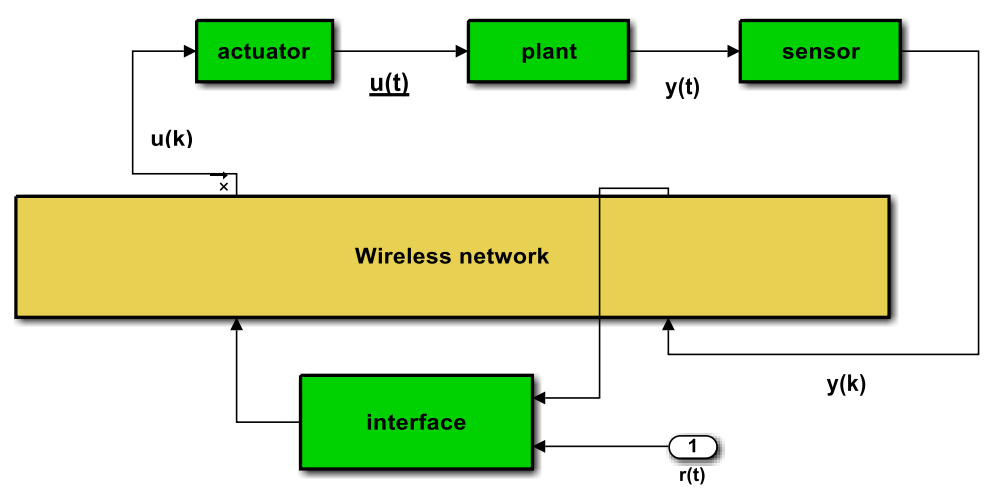

Figure 2. WNCS design 
These characteristics have to be identified and understood for enhancing the process of systems design for effective working and assuring the ability of WNCS to achieve specific useful tasks. These characteristics include a link quality time delays, reliability, jitter, scalability, security, energy consumption, the distance between nodes, flexibility and control paradigms [30].

There is a trade-off between communication and control performances. Therefore, wireless communication protocols and theories of a control system must be designed collaboratively.

As can be seen in Figure 2, $y(t)$ is a plant/process where its result is identified by the sensor (S). Additionally, the delay time for sampling and decoding has a delay time $(\tau \mathrm{s})$.

Over the wireless network, it is transmitted to the Controller (C) from the client side. For packets transmission, both switching function $(\beta)$ and delay time $(\tau c)$ are considered. In the beginning, a time will be requested for processing by the signal at the client side. This time can be considered as a delay time ( $\tau \mathrm{sc})$. Next, there will be a control signal (uk). This signal will be transmitted along the exact wireless network. The switching function is symbolized by $(\alpha)$ and the time delay is $(\tau \mathrm{ca})$. Thereby, the overall time is given by.

$$
\mathrm{t}=\tau_{k}^{S}+\tau_{k}^{\mathrm{sc}}+\tau_{k}^{c}+\tau_{k}^{\mathrm{ca}}+\tau_{k}^{\mathrm{sa}}
$$

Here, $\mathrm{t}$ has to be less than $\mathrm{h}$ (the sampling interval time) in order to obtain proper operation of the wireless networked control system.

Specifically, a new control signal (uk) cannot be calculated by the controller unless a new sensor measurement $\mathrm{y}^{\wedge}$ it receives. Then, the old control signal $\mathrm{u}^{\wedge}$ is still used by the actuator to actuate the plant/process to the acquired operation state. In consequence, we can formulate the estimated output and input as follows:

$$
\begin{aligned}
& \hat{y}=\left(1-\beta_{k}\right) y_{k} \\
& \hat{u}=\left(1-\alpha_{k}\right) u_{k}
\end{aligned}
$$

In a wireless network, the switching functions stand for the probability of the succeeded arrival of $\mathrm{y}^{\wedge}$ and $\mathrm{u}$. These parameters are symbolized as $\rho_{k}^{\mathrm{sc}}, \rho_{k}^{\mathrm{ca}}$ with assuming that:

$$
\rho_{k}^{\mathrm{sc}}, \rho_{k}^{\mathrm{ca}}=\left\{\begin{array}{l}
1 \text { data lost } \\
0 \text { data received }
\end{array}\right.
$$

$\tau_{\mathrm{k}}$ and $\rho_{\mathrm{k}}$ represent network parameters which vary based on the chosen node hardware, medium access control and network traffic. The state space model for the process/plant (which consists of the mentioned parameters) can be formulated as below.

$$
x(k+1)=\phi(h) X(k)+\Gamma_{0}\left(h, \tau_{k}\right) u+\Gamma_{1}\left(h, \tau_{k}\right)
$$

Thus, the predicted yields to the plant is as follows:

$$
\hat{y}(k)=\rho_{k}^{s c}(C x(k))
$$

At the client yield, the predicted control signal $\mathrm{u}_{-} \mathrm{k}$ can be formulated as:

$$
\hat{u}_{k}=\rho_{k}^{s c} \rho_{k}^{c a} u_{k}+\left(1-\rho_{k}^{s c} \rho_{k}^{c a}\right) \hat{u}_{k-1}
$$

where the explanation of $\Phi, \Gamma 0, \Gamma 1$ is examined in various WCN. The purpose of the controller is based on the state-space model, and it is formulated by:

$$
\hat{u}_{k}=K(h, \tau, \rho)\left[\begin{array}{c}
x_{k} \\
\hat{u}_{k-1}
\end{array}\right]
$$

Figure 3 shows the NWCS block diagram with the essential parameters that influence total system performance and the network. 


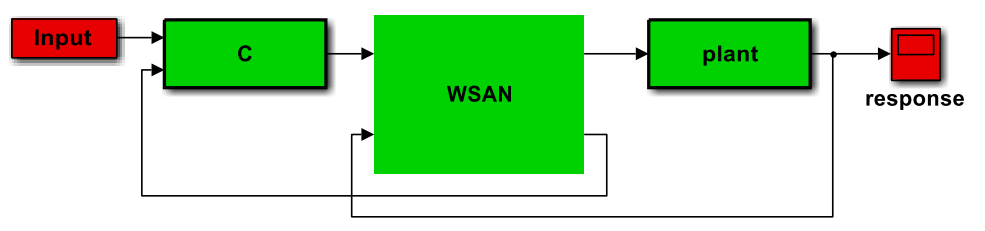

Figure 3. Block diagram of the WNCS

After 1915s in the last century, significant interest was directed to using digital computers as a tool for closed-loop control. The control designs transited from the continuous-time/continuous-states models to the discrete-time/quantized-states models of digital control. Usually, the continuous-time/continuous-states models used in conventional feedback designs. When designing digital control, a number of new issues like selection of sampling period, the finite length of the word, the resulting effects of quantization, resources of memory and bounded computational must be considered with focusing on their impacts on the control performance.

Also, remote monitoring systems and remote data-acquisition systems can be considered in this type of systems. A local site is typically defined as the area where a central controller is set; while a remote site is an area where the plant is situated, which is comparable to the client-server computer network scheme. The client-server control system platform architecture comprises two terminal computers. One client is used for performing the control algorithms like (PID), and the other one for plant data acquisition and interfacing. This operation is illustrated in Figure 4.
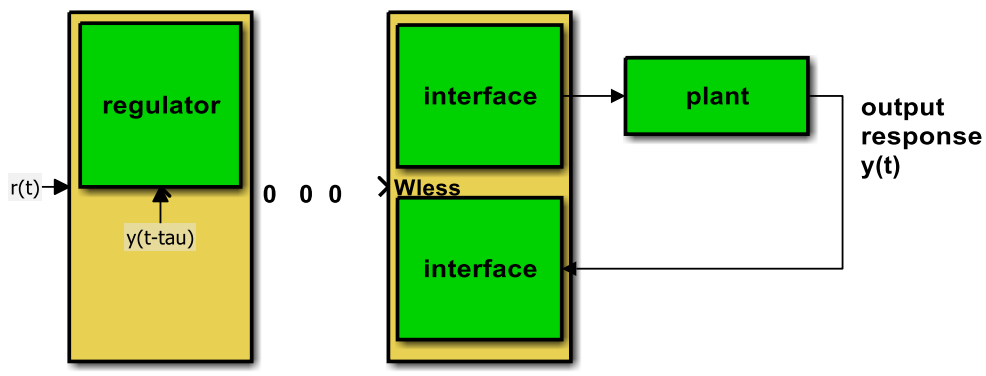

Figure 4. The Fly-Wheel schematic diagram (server)

Let's come to the communication network, the two PCs can be linked either by wire or wireless. Such a scheme is used for connecting the components of control systems through TCP/IP or User Datagram Protocol (UDP) of the Internet for the implementation of remote control [31].

The controller and the plant can be connected by using a Wireless Sensor and Actuator (WSAN). The output of plant measurement from sensors which (i.e. $y(t)$ ) is sent over wireless workstation to the controller. Whereas, over other wireless workstations, the control signal is transmitted to the actuators.

\subsection{Experimental Setup}

The hardware devices of the wireless position control system consist of three mains section as follows: plant, controller and wireless sensor network devices as shown in Figure 5.

As shown in Figure 5, a National Instruments (NI USB-6008) interfacing card to link the PC with the plant. The connection between the position control system (F-W PC.S.) and the workstation is performed through this card at the server side. The NI-max represents the software, which is employed to drive the NI card. This software interfaces the Simulink blocks of the DAQ toolbox and the plant. The interface with the XBee platform is performed through the Simulink blocks from Instrument Control Toolbox like serial transmit/receive. 


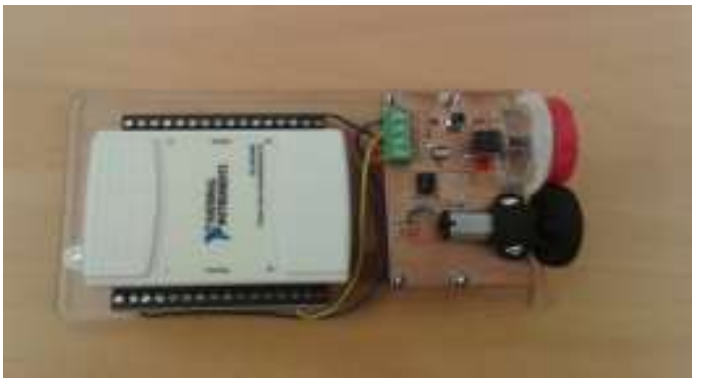

Figure 5. F-W PCS Test-bed screenshot

The NI card is used to interface the plant with the workstation using MATLAB 2018, after completing the hardware system design. It is well known that the MATLAB/Simulink simulation environment consists of various powerful tools capable of simulating any control system including WNCSs. It is worth mentioning that the WNCS simulation strategy utilizing a specific remote servo control system is explained.

The transfer function of the plant model can be formulated as follows:

$$
\frac{\theta_{1}}{v_{a}}=\frac{43}{\mathrm{~s}(1.127 \mathrm{~s}+1))} \mathrm{e}^{-\mathrm{s}}
$$

Utilizing the system identification toolbox of MATLAB, we can estimate the transfer function from the measured information of the input/output (i.e., voltage - angle) of the F-WPCS.

Figures 6 and 7 illustrate the input information (voltage) to the system and the output information (angular position), respectively.

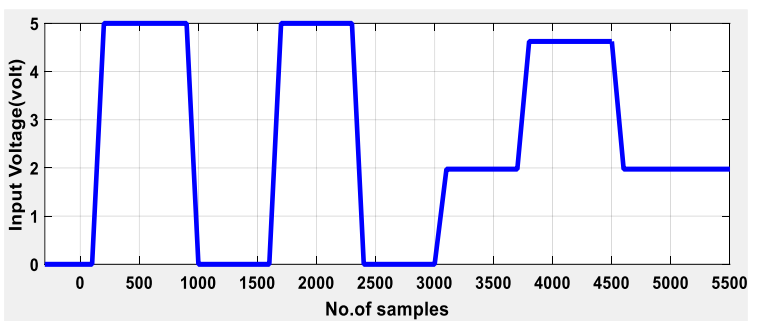

Figure 6. Input information to the (F-W PCS)

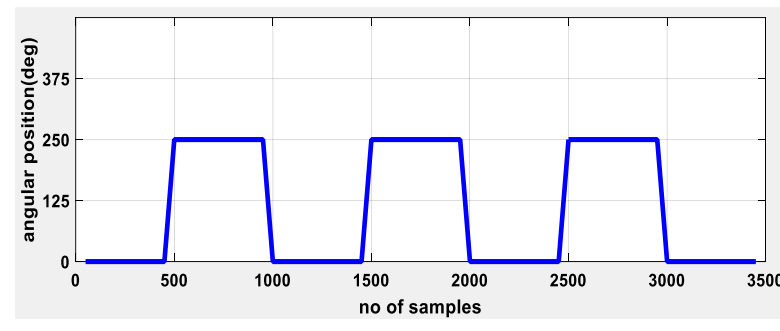

Figure 7. Output information from the system

Additionally, Figure 8 clarifies the practical research topic application. Now we completed allocating the system model, the HW/SW connection and software design of the position control system. Thus, a (PID) controller was designed to amend the system to follow up the reference input.

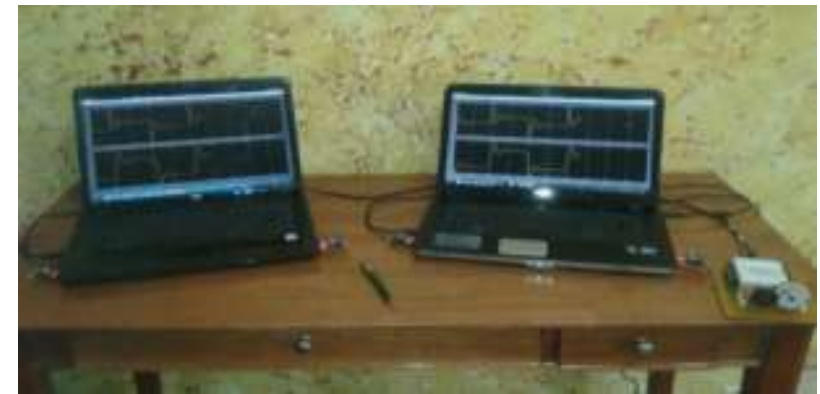

Figure 8. Wireless control system for (F-W PCS) 


\section{PROPOSED ALGORITHM IMPACT}

The course from which the students were involved was a control engineering course for undergraduate students in the fourth year in the electrical engineering department at the University of Tikrit. To predict the effectiveness of the Wi-Fi project on students' learning and intellectual capacity, the class was given MCQ on control engineering" in two tests: the first test was before introducing the projects and the second test after the entire class has a chance to obtain hands-on skill with the Wi-Fi project. Both tests were conducted to measure any change in student's learning and comprehension.

The class capacity is 20 students, 11 male and 9 female. The exam consisted of 12 MCQs covering various aspects of engineering control. The test consists of $12 \mathrm{MCQ}$. The percentage of answers before and after the project is shown in Figure 9. As demonstrated in Figure 10, progress is shown in the whole class on each of the (12) questions. Such progression can be counted by the practical experience that the students obtained from the (Wi-Fi) project between the two exams since no other types of orders were presented to the students before the second test. The pre/post exams were conducted among students with an exact background and who had offered to the exact theoretical subjects.

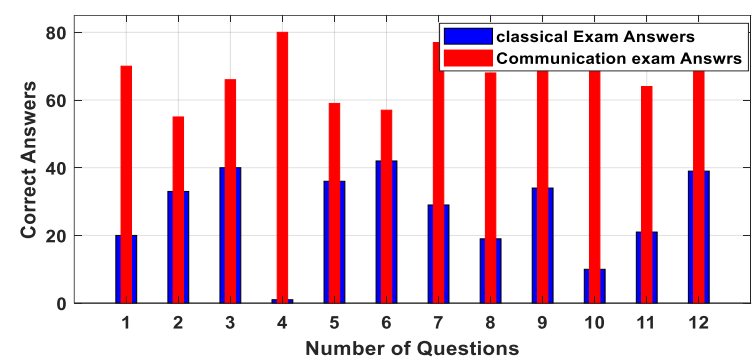

Figure 9. Wireless control system for F-W PCS

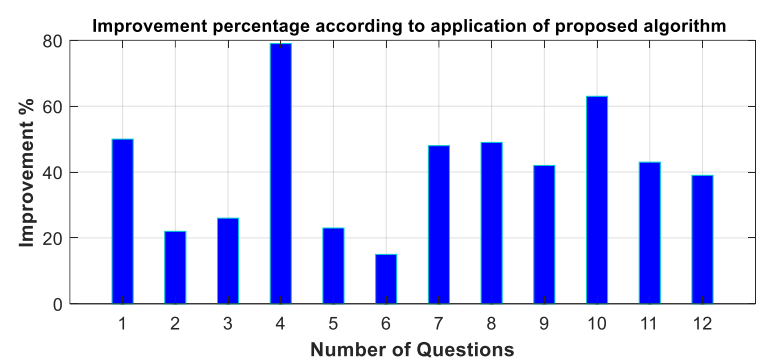

Figure 10. Wireless control system for F-W PCS.

\section{CONCLUSION}

In this study, we proposed a new e-learning model aims to encourage and enhance the level of students in various learning purposes and stages. The results demonstrate the feasibility of implementing the feedback control system over a wireless sensor network in order to link both the controller and the plant remotely. It is clear that the PID parameters can be easily tuned in case of inducing of both delay and packet loss of the wireless network. This tuning may control system stability and performance. The students' responses showed favourable reactions to the project demonstrations. The responses indicate that the Wi-Fi projects are so helpful and easy to implement in the understanding of course subjects; thereby improvement in learning and comprehension.

Finally, the experiments have proved that the designing of the client-server scheme using MATLAB is more efficient in developing the control strategy and plant interfacing with the NI-6008 card.

\section{REFERENCES}

[1] A. Habibi.A. Mukminin,Y. Riyanto ,L. Diat Prassojo ,U. Sulistiyo M. Sofwan, F, Saudagar, "Building An Online Community: Student Teachers Perceptions on The Advantages of Using Social Networking Services in A Teacher Education Program", Turkish Online Journal of Distance Education-TOJDE, vol. 19, no. 1 Article 4, pp.46-61, 2018.

[2] J. B Son, "Technology and Language Teacher Education In: Teacher Development in Technology-Enhanced Language Teaching", Palgrave Macmillan, Cham, 2018.

[3] I. N. Sarkar, C. M. Trevor, "Teaching Wireless Communication and Networking Fundamentals Using Wi-Fi Projects", IEEE Transactions on Education, vol. 49, no. 1, pp.98-104, 2006.

[4] C. Russell, Malfroy, J., Gosper, M., \& McKenzie, J., "Using research to inform learning technology practice and policy: A qualitative analysis of student perspectives", Australian Journal of Educational Technology, vol. 30, no. 1, pp.1-15, 2014.

[5] J. Hespanha , P. Naghshtabrizi and Xu Y., "A survey on recent results in networked control systems," in Proceedings of IEEE, vol. 95, no. 1, 2007.

[6] Product Catalogue, "Innovative applications for wireless control and telemetry", www.remotecontroltech.com, 2013.

[7] Cao X., Chen Y., Xiao Y. and Sun Y., "Control system design for wireless sensor and actuator networks," IEEE Communication Society Proceedings, 2008. 
[8] Musaria K. Mahmood, Fawzi M. Al-Naima, and N. K. Uzunoglu, "Design and simulation of a data transmission network for industrial control system subject to reliability improvement”, Intern. Conf. on Future Communication Networks (ICFCN'12), Baghdad, 2012.

[9] A. Nayak, and I. Stojmenovic, "Wireless sensor and actuator networks", John-Whiley \& sons, 2010.

[10] S. Zampieri, "Trends in networked control systems", in the Proceedings of the 17th. World Congress International Federation of Automatic Control, Korea, 2008.

[11] G. Nikolakopoulos, "Lecture notes on industrial automation and process control", Department of Computer Science, Electrical and Space Engineering, LTU, Sweden, 2013.

[12] G. Nikolakopoulos, A. Panousopoulou, Tsez A. and Lygros J., "Multi-hopping induced gain scheduling for wireless networked control systems", The Asian Journal of Control, vol. 9, no. 4, pp.450-457, 2007.

[13] L. Dritsas , G. Nikolakopoulos and Tzes A., "On the modelling of networked control systems", in the Proceedings of the Mediterranean Conference on Control and Automation, Greece, 2007.

[14] Nikolakopoulos G., A. Panousopoulou, and Tsez A., "Experimental controller tuning and QoS optimization of a wireless transmission scheme for real-time remote control applications", Elsevier,pp. 333-346, 2008.

[15] Y. Uchimura , "Wireless network based identification and control with variable time delay", The IEEE Proceedings, pp. 336-341, 2008.

[16] S. Dai, Lin H. and Ge S., "A switched system approach to scheduling of networked control systems with communication constraints", The 48th IEEE Conference on Decision and Control, pp.4991-4996, China, Dec. 2009.

[17] G. Nikolakopoulos, Alexis K., Arvanitakis I. and Tsez A., "A collaborative unmanned helicopter control strategy for image compression and wireless transmission", in the 81st Mediterranean Conference on Control and Automation, pp.1206-1211, 2010.

[18] X. Li, S. Munigalas and Zeng Q., "Design and implementation of a wireless programmable logic controller system", in the International Conference on Electrical and Control Engineering, pp.3138-3141, USA, 2010.

[19] A, Bemporad. Di Cairano S., Henrikson E. and Johanson K., " Hybrid model predictive control based on wireless sensor feedback, an experimental study", in The International Journal of Robust and Nonlinear Control, pp.209-225, 2010.

[20] O. Kaltiokallio, M. Eriksson and M. Bocca, "On the performance of the PIDPLUS controller in wireless control systems", in the 18th Mediterranean Conference on Control Automation, pp.707-714, Spain, 2010.

[21] M. Iacob, G. Andreescu, and N. Muntean, "SCADA System for a central heating and power plant", 5th Int. Symp. on Applied Computer Intelligent and Informatics, Romania, pp. 159-164,2009.

[22] Park P., Araujo J. and Johanson K., "Wireless networked control system: co-design", in International Conference on Networking, Sensing and Control, Netherland, 2011.

[23] Lemmon M. and Hu X.," Almost sure stability of wireless networked control systems under exponentially bursts of drop-outs," in $H S C C^{\prime} 11$, pp.12-14, USA, 2011.

[24] Choi R., Lee S. Lee D. and Yoo L., "Packet loss compensation for cyber-physical control systems", in IEEE/ACM Third International Conference on Cyber-physical Systems, 2012.

[25] Qu F., Guan Z., Yauan F. and Zhan X., "Stabilization of wireless networked control systems with packet loss and impulse disturbance", in the Proceedings of 10th World Congress of Intelligent Control and Automation, China, July, pp.1031-1036, 2012.

[26] M. K. Mahmood, and F. Al-Naima, "An Internet Based Distributed Control Systems: A Case Study of Oil Refineries”, Energy and Power Engineerings, pp. 310-316, 2011.

[27] S. Ghavifekr \& W.A.W Rosdy, "Teaching and learning with technology: Effectiveness of ICT integration in schools", International Journal of Research in Education and Science (IJRES), vol.1, no.2, pp. 175-191,2015.

[28] https://annhayesbell.org/spreading-the-news-new-computer-science-framework-and-iste-students-standards/

[29] Amarawardhana et al., "Case study of WSN as a replacement for SCADA", the 4th International Conference on Industrial and Information Systems (ICIIS), SriLanka , pp.49-54, 2009.

[30] Ulusoy A., Onat A. and Gurbuz O., "Wireless model predictive networked control system over IEEE802.15.4", the 2013 EEE International Conference on Distributed Computing Sensor Systems, pp. 382-387, 2013.

[31] S. Hamid, S. Kurnia, J. Waycott \& S. Chang, "Understanding students' perceptions of the benefits of online social networking use for teaching and learning", Internet and Higher Education, pp. 1-9, 2015.

\section{BIOGRAPHY OF AUTHORS}

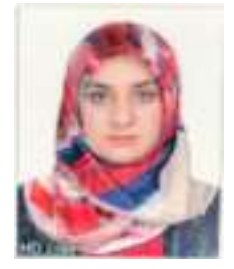

Nada Nasih Tawfeeq received her BSc.in ElectricalEngineering from Tikrit University in 2009 and MSc from Mosul University with communication specialization in 2014. Her research area interest includes antenna, digital signal processing and wireless communication system design. Currently she is working as an Assistant Instructor in university ofTikrit/College of Engineering/Electrical department. 


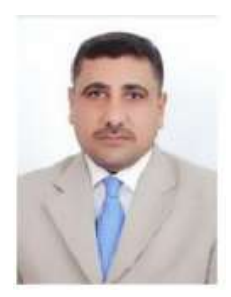

Mehdi J. Marie was born in Baghdad, Iraq in 1970. He received his Bachelor's (1993), Master's (2004) Degrees from University of Technology (Iraq) and Ph. D. from the University of Basrah (2014). He has been a lecturer of Control Theory I, II, Electronics and Electrical Networks at the Al-Nahrain University, College of Engineering. He is currently a senior engineer at Al-Zawaraa State Company, Ministry of Industry and Minerals (Iraq). Dr. Mehdi had achieved over seven Journal articles and two conference papers in the field of control and systems engineering. His current research and development interests are mainly in the following areas: self-tuning control and system identification, controller design for linear and non-linear systems, industrial applications particularly in the processing industry (for example refining, cement, plastic and food industry).

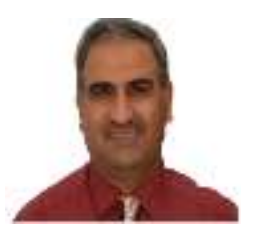

Khalaf S.Gaeid received the B.Sc. degree in electrical engineering/Control from MEC,Baghdad, Iraq in 1993 and the M.Sc degree in Control Engineering from University of Technology, Baghdad, Iraq in 2004. He graduated from University of Malaya, Malaysia in 2012 with the PhD degree in control system and machine drives. His doctoral researchwas in the development new fault tolerant controller based wavelet for induction machines.He is currently assistant professor in control systems at the Department of Electrical Engineering, College of Engineering, Tikrit University, Tikrit, Iraq. His research interests include fault tolerant control, wavelet, fault diagnosis,machine drives and their applications in electrical engineering.He is IEEE member with a lot of publications in high rank journals and confrences. 